\title{
Reliability-based Robust Design of Smart Sensing Systems for Failure Diagnostics Using Piezoelectric Materials
}

\author{
Pingfeng Wang ${ }^{1}$, Zequn Wang ${ }^{2}$, Byeng D. Youn ${ }^{3}$ and Soobum Lee ${ }^{4}$ \\ ${ }^{1,2}$ Department of Industrial and Manufacturing Engineering, Wichita State University \\ Wichita, KS 67260, USA \\ ${ }^{3}$ School of Mechanical Engineering, Seoul National University \\ Seoul, South Korea \\ ${ }^{4}$ Department of Mechanical Engineering, University of Maryland \\ Baltimore County, MD21250, USA
}

\begin{abstract}
This paper presents a reliability-based robust design approach to develop piezoelectric materials based structural sensing systems for failure diagnostics and prognostics. A detectability measure is defined to evaluate the performance of any given sensing system, and the sensing system design problem can be formulated to maximize detectability for different failure modes by optimally allocating piezoelectric materials into a target structure. This formulation can be conveniently solved within a reliability-based robust design framework to ensure design robustness while considering the uncertainties such as those from structure properties and operation conditions. Two case studies, that design sensor networks for an aircraft wing panel and a power transformer structure, are employed to demonstrate the effectiveness of the proposed methodology in developing multifunctional material sensing systems.
\end{abstract}

${ }_{1}^{1}$ Assistant Professor, pingfeng.wang@ wichita.edu, Corresponding Author

${ }^{2}$ Graduate Student, zxwang5@wichita.edu

${ }^{3}$ Associate Professor, bdyoun@snu.ac.kr

${ }^{4}$ Assistant Professor, sblee@umbc.edu 


\section{Nomenclature}

$\begin{array}{lll}R & = & \text { reliability } \\ \Phi & = & \text { standard Gaussian cumulative distribution function } \\ \beta_{\mathrm{t}} & = & \text { target reliability index } \\ C_{L} & = & \text { user-defined confidence level } \\ F_{(x)} & = & \text { cumulative distribution function } \\ F^{-1}(x) & = & \text { inverse cumulative distribution function } \\ f_{x}(x) & = & \text { probability density function } \\ f_{(\cdot \mid \cdot)} & =\text { conditional probability density function or likelihood function } \\ p_{f s} & =\text { probability of system failure } \\ G_{i} & =\quad \text { function of the } i^{t h} \text { constraint } \\ C & \end{array}$

\section{Introduction}

With a growing complexity of engineered systems, failure diagnostics techniques have been prevalently employed to prevent potential catastrophic failures and improve system reliability and safety. Real-time health diagnostics interpret data acquired by smart sensors, and utilize these data streams in making critical operation and maintenance decisions [1]. Enormous benefits can be provided by effective health diagnostics activities, such as improved system safety, reliability, and reduced costs for the operation and maintenance of complex engineered systems. Structure maintenance and life-cycle management is an area that can significantly benefit from diagnostics and improved maintenance practices, as unexpected system breakdowns could be prohibitively expensive [2]. Thus to reduce and possibly eliminate such problems, it is important to accurately assess the health condition of an operating system in real time through effective health diagnostics. Researches on condition monitoring address these challenges by assessing system health states utilizing sensory information from the functioning system [3-5]. Monitoring of system health state (HS) changes over time provides valuable information about the performance degradation of system components for critical maintenance decision makings, and has been successfully applied to 
many engineering systems such as bearings [6-9], machine tools [10], power transformers [11], engines [12], aircraft wings [13], and turbines [14]. In the literature, there are two categories of approaches in general that are often employed for health diagnostics, machine learning techniques and statistical inference techniques. The machine learning-based health diagnostics approaches can further be divided into supervised learning, unsupervised learning and semi-supervised learning techniques. In addition to the aforementioned machine learning-based algorithms, statistical inference-based algorithms can also be used to classify system HSs based on statistical distances such as Mahalanobis distance [15], k-nearest neighbor method [16] and k-mean clustering [17]. Significant advancements in diagnostics area have been achieved by applying classification techniques based on machine learning or statistical inferences, resulting in a number of classification methods, such as back-propagation neural networks [18-21], deep belief networks [22, 23], support vector machines [24-28], self-organizing maps [29], and Mahalanobis distance (MD) [15]. Some researchers combined two or more existing techniques to form hybrid models to achieve better diagnostic performance. Zhang et al. [9] proposed a bearing fault diagnosis methodology using multi-scale entropy (MSE) and adaptive neuro-fuzzy inference system. Saimurugan et al. [24] presented a multi-component fault diagnosis of a rotational mechanical system based on decision trees and support vector machines.

Despite a variety of numerical diagnostics algorithms being developed and a broad range of successful applications in various engineering fields being reported in the literature, one of the key challenges in structural health diagnostics lies in the fact that health relevant sensory data must be collected effectively so that enough evidences can be provided for diagnostics algorithms to conduct health state identification and damage detection. However, implicit relationship between sensory signals and system health states as well as sensor noise and uncertainties related to system operating conditions render a grand challenge in developing an effective sensor network so that system health states can be accordingly diagnosed accurately with sensory data collected from the sensor network. To overcome this challenge, a sensor network must be developed with sensors nodes being optimally placed so that the differences between different system health states can be reflected clearly on sensory signals. In addition, the sensor network must be designed to ensure the robustness of health diagnostics given the aforementioned uncertainties and variability involved in sensing and diagnosing processes. In the literature, sensor placement optimization under uncertainties has been studied for structural health monitoring applications [30, 31], and further optimal location of sensors has been presented for parametric identification of linear structural systems [32]. A methodology for optimally locating sensors in a dynamic system $[33,34]$ was developed as a probabilistic 
approach in structural health monitoring system. The study in [35] developed a Bayesian approach to optimize sensor placement for structural health monitoring. In [36], an optimal sensor location methodology for structural identification and damage detection is studied. Most of these methods were settled for allocating a number of sensors to distinguish a specific health state of structural damage, and their applications were limited by the type of health state failure mechanisms. Although reported studies on sensor placement optimization have showed improvements on health diagnostics performance, there are two fundamental challenges that hinder the broad applications of this technique. First, the sensing capability of the sensor nodes used in sensor placement studies have been mostly assumed to be independent to the target systems, which is generally not true for practical structural applications; Second, there is no quantitative measure for the diagnostics performance related based upon a given sensor network design, thus, the performance robustness cannot be ensured in the sensor network design process.

To address the aforementioned sensor network design challenges for structural diagnostics applications, this paper presents a novel reliability-based robust design optimization (RBRDO) framework for structural sensing function design using multifunctional materials. The RBRDO technique has been developed to ensure the performance robustness thus improve quality and reliability in product and process design, while considering uncertainties involved in different stages of a system's life cycle [37-42]. In detail, design optimization of piezoelectric embedded sensor patches is considered to realize structural sensing function [43-48]. First, a generic detectability measure is defined in this study to quantify the performance of a given sensing system for diagnostics under uncertainty. A novel detectability analysis approach based on Mahalanobis distance classifier is then developed to carry out the detectability analysis for a given sensing system design. Second, with the defined detectability measure and developed detectability analysis approach, a novel reliability-based robust design optimization (RBRDO) framework is presented for sensing system design in order to minimize the system development costs while maintaining the predefined detectability target. The rest of the paper is organized as follows. First, smart sensing with piezoelectric materials is introduced in section 2. In section 3, a detectability measure is defined in a probabilistic form as a unified quantitative measure for the performance of any given sensing system used for the structural health diagnostics. A general approach for detectability evaluation is also introduced based on health state classification. In section 4, a generic RBRDO framework is developed to design smart material systems for the structural health diagnostics and prognostics. Two case studies are used in section 5 to demonstrate effectiveness of the proposed methodology in developing structural sensing system design methodology. 


\section{Smart Sensing with Piezoelectric Materials}

Piezoelectric materials can be potentially applied in both sensing and actuating applications [49-53]. In sensing applications the PZT sensor is attached to a structure and exposed to a stress field that creates electric charges (direct piezoelectric effect). In actuating applications the PZT actuator is attached to a structure and an external electric source is applied to the actuator that induce strain field (reverse piezoelectric effect). In both cases the constitutive relationship can be mathematically formulated as follows:

$$
\begin{aligned}
& \varepsilon_{i}=S_{i j}^{E} \sigma_{j}+d_{m i} E_{m} \\
& D_{m}=d_{m i} \sigma_{i}+e_{i k}^{\sigma} E_{k}
\end{aligned}
$$

where the indexes $i, j=1,2, \ldots, 6$ and $m, k=1,2,3$ refer to different directions within the material coordinate system [51], $\sigma$ is a vector of the stress $(\mathrm{N} / \mathrm{m} 2)$ and $\varepsilon$ is a vector of the strain, $d$ is a matrix of the piezoelectric strain constants that defines strain per unit at constant stress $(\mathrm{m} / \mathrm{V}), E$ is a vector of the electric field $(\mathrm{V} / \mathrm{m}), S^{E}$ is a matrix of the elastic compliance $\left(\mathrm{m}^{2} / \mathrm{N}\right), D$ is a vector of the electric displacement (Coulomb/m2), $e^{\sigma}$ is a matrix of the dielectric permittivity $(\mathrm{F} / \mathrm{m})$. This paper considers a sensing application which utilizes $d_{31}$ effect of PZT material: a voltage output along the thickness direction as a response of in-plane strain (see Figure 1).

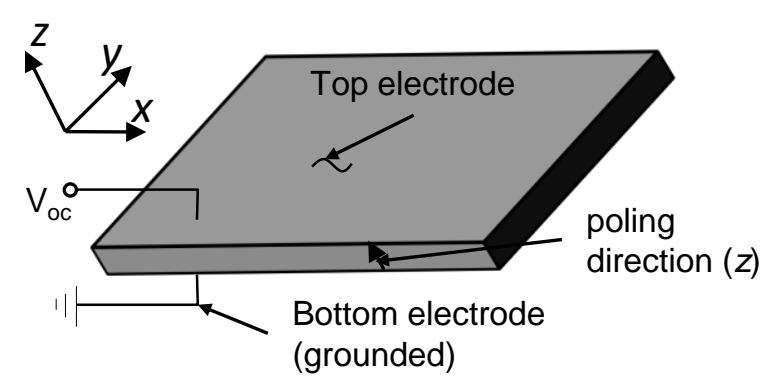

Figure 1: Schematic of a piezoelectric ceramic sheet

This paper considers use of piezoelectric materials for real-time monitoring of structural damages utilizing direct piezoelectric effect - generation of electric potential in response to an applied mechanical stress. A harmonic vibration with a fixed frequency is assumed for a structure to be monitored. Harmonic response analysis solves the time-dependent equation of motion for linear structures under steady-state vibration with a fixed excitation frequency. Considering a general equation of motion for a piezoelectric coupled-field structure after the application of the variational principle and finite element (FE) discretization, the coupled FE matrix equation is derived as [54]: 


$$
\left[\begin{array}{cc}
\mathbf{M} & \mathbf{0} \\
\mathbf{0} & \mathbf{0}
\end{array}\right]\left\{\begin{array}{c}
\ddot{u} \\
\ddot{V}
\end{array}\right\}+\left[\begin{array}{cc}
\mathbf{C} & \mathbf{0} \\
\mathbf{0} & \mathbf{0}
\end{array}\right]\left\{\begin{array}{c}
\dot{u} \\
\dot{V}
\end{array}\right\}+\left[\begin{array}{cc}
\mathbf{K} & \mathbf{K}^{z} \\
\left(\mathbf{K}^{z}\right)^{\mathbf{T}} & \mathbf{K}^{d}
\end{array}\right]\left\{\begin{array}{c}
u \\
V
\end{array}\right\}=\left\{\begin{array}{c}
F \\
L
\end{array}\right\}
$$

where $\mathbf{M}, \mathbf{C}$, and $\mathbf{K}$ are the structural mass, damping, and stiffness matrices respectively, $\mathbf{K}^{\mathbf{z}}$ is piezoelectric coupling matrix, $\mathbf{K}^{\mathbf{d}}$ is dielectric conductivity matrix, $\{u\}$ is displacement vector, $\{V\}$ is voltage vector, and $\{F\}$ and $\{L\}$ are structural and electrical load vectors. The electrical load vector $\{L\}$ is assumed to be zero in this paper, thus, only the structural input loading exists for the design of structural sensing functions. Although the application problems with piezoelectric material patches could have very little effect on the structure stiffness, however, it could still affect the PZT sensor outputs. Thus, in the presented sensor network design study, the coupling effects have been taken into consideration, as shown in Eq. (3), in which $\mathbf{K}^{\mathbf{z}}$ indicates piezoelectric coupling matrix. In the harmonic response analysis, all points in the structure are vibrating at a same known frequency. Therefore displacements and voltage can be defined as:

$$
\begin{aligned}
& \{u\}=\left\{u_{\max }\right\} e^{j(\varphi+\omega t)}=\left\{u_{\max }(\cos \varphi+j \sin \varphi)\right\} e^{j \omega t}=\left[\left\{u_{r e}\right\}+j\left\{u_{i m}\right\}\right] e^{j \omega t} \\
& \{V\}=\left\{V_{\max }\right\} e^{j(\varphi+\omega t)}=\left\{V_{\text {max }}(\cos \varphi+j \sin \varphi)\right\} e^{j \omega t}=\left[\left\{V_{r e}\right\}+j\left\{V_{i m}\right\}\right] e^{j \omega t}
\end{aligned}
$$

where $\left\{u_{\max }\right\}$ and $\left\{V_{\max }\right\}$ are the maximum displacement and voltage, respectively; $\varphi$ is the phase angle, $\omega$ is the imposed excitation frequency, $j$ is the square root of -1 , and the sub-indices $r e$ and $i m$ represent the real and imaginary components, respectively. The force vector can be specified analogously as follows:

$$
\{F\}=\left\{F_{\max }\right\} e^{j(\varphi+\omega t)}=\left\{F_{\max }(\cos \varphi+j \sin \varphi)\right\} e^{j \omega t}=\left[\left\{F_{r e}\right\}+j\left\{F_{i m}\right\}\right] e^{j \omega t}
$$

Finally, substituting Eq. (4) and Eq. (5) into Eq. (3) and cancelling the $e^{j \omega t}$ term, the following structural equations [54] can be obtained

$$
\left(-\omega^{2}\left[\begin{array}{cc}
\mathbf{M} & \mathbf{0} \\
\mathbf{0} & \mathbf{0}
\end{array}\right]+j \omega\left[\begin{array}{cc}
\mathbf{C} & \mathbf{0} \\
\mathbf{0} & \mathbf{0}
\end{array}\right]+\left[\begin{array}{cc}
\mathbf{K} & \mathbf{K}^{z} \\
\left(\mathbf{K}^{z}\right)^{\mathrm{T}} & \mathbf{K}^{d}
\end{array}\right]\right)\left[\begin{array}{l}
\left\{u_{r e}\right\}+j\left\{u_{i m}\right\} \\
\left\{V_{r e}\right\}+j\left\{V_{i m}\right\}
\end{array}\right]=\left[\begin{array}{c}
\left\{F_{r e}\right\}+j\left\{F_{i m}\right\} \\
\mathbf{0}
\end{array}\right]
$$

One of the commonly used piezoelectric materials is lead zirconate titanate (PZT), a piezoelectric ceramic, which has wide application in vibration sensors and health monitoring systems [46]: a self-sensing piezoelectric actuator for collocated control [47], health monitoring/damage detection of a rotorcraft planetary gear train system using piezoelectric sensors [48], and smart sensor system for structural condition monitoring of wind turbines [49]. In this paper a new smart sensing system design framework will be developed for structural health diagnostics using piezoelectric materials. 


\section{Probabilistic Detectability Measure for Diagnostics}

Sensing systems can monitor the physical behaviors and determine the health state of a system. However, false alarm may occur due to the uncertainties of system operation processes and manufacturing. Therefore, the performance of sensing system should be qualified using a probabilistic method, so that the accurate detection of system health states can be achieved. In the proposed RBRDO framework, a set of health states must be categorized based on historical failure data. Thus correct and incorrect detection rates of every health state can be defined as the sensing performance measures. The correct detection rate can be calculated by a conditional probability that the sensing system can correctly detect the health state given that the system is operated at one health state. In contrast, the incorrect detection rate can be expressed as a conditional probability that the sensing system detects wrong information. Using the data of correct and incorrect detection rates, the sensing system detectability can be expressed by constructing a probability-of-detection $(\mathrm{PoD})$ matrix.

This section introduces the concept of detectability and the method to evaluate the detectability for each health state based on structural simulation and system health state classification. Subsection 3.1 introduces the concept of probability of detection matrix to qualify the sensing system. Subsection 3.2 demonstrates the usefulness of probability of detection using a mathematical example. Subsection 3.3 presents the Mahalanobis distance based approach for health state classification. In this research, it is assumed that all numerical models are valid and they deliver accurate results associated with actual systems.

\subsection{Probability-of-detection (PoD) matrix}

A PoD matrix defines the overall sensing system diagnostics performances, which consists of the probabilities of correct detection and misdetection for all predefined health states. A general form of the PoD matrix is shown as

$$
\mathrm{PoD}=\left[\begin{array}{ccccc}
P_{11} & P_{12} & P_{13} & \cdots & P_{1 N_{H S}} \\
P_{21} & P_{22} & P_{23} & \cdots & P_{2 N_{H S}} \\
P_{31} & P_{32} & P_{33} & \ldots & P_{3 N_{H S}} \\
\vdots & \vdots & \vdots & \ddots & \vdots \\
P_{N_{H S} 1} & P_{N_{H S} 2} & P_{N_{H S} 3} & \ldots & P_{N_{H S} N_{H S}}
\end{array}\right]
$$

In the PoD matrix, $N_{H S}$ represents the number of health states $(H S)$, where $P_{i j}$ indicates the probabilistic relationship between the true system health state, $i$, and the health state, $j$, detected by the sensing system. $P_{i j}$ can be defined as 
the conditional probability that the system is detected to be operated at $H S_{j}$ by the sensing system given that the system is operated at $H S_{i}$. This relationship can be statistically expressed as

$$
P_{i j}=\operatorname{Pr}\left(H S_{j} \text { as Detected } \mid H S_{i} \text { as True }\right)
$$

To provide a probabilistic measure for diagnostics performance of a sensing system while considering uncertainties induced in manufacturing and system operating processes, detectability can be defined for each system health state accordingly based on the diagonal terms in the PoD matrix. As an example, the detectability for the $i^{\text {th }}$ system health state $\left(H S_{i}\right)$ can be statistically defined below as

$$
D_{i}=P_{i i}=\operatorname{Pr}\left(H S_{i} \text { as Detected } \mid H S_{i} \text { as True }\right)
$$

While considering all health states, the diagonal terms in the PoD matrix, which represent the probabilities of correct detection for predefined health states, will determine the overall SN detection performance. With the predefined detectability requirements, these diagonal terms in the PoD matrix will then constitute $N_{H S}$ number detectability targets to be satisfied during the sensing function design process. In the following, an example is employed to demonstrate the proposed detectability measure.

Suppose that only one sensor is used to collect data for diagnostics and three predefined health states are given as following: (1) Health State $1\left(H S_{l}\right)$ : the system is operating normally without any damage and sensor output follows a normal distribution as $N\left(1,0.9^{2}\right)$; (2) Health State $2\left(H S_{2}\right)$ : the system is operating but has some minor damage that follows a normal distribution as $N\left(3,0.6^{2}\right)$ and (3) Health State $3\left(H S_{3}\right)$ : The system is operating but has a severe damage that follows a normal distribution as $N\left(6,1.5^{2}\right)$. In what follows, the detectability values for all three defined health states will be determined based on the available information. To calculate the detectability value for each health state, it is necessary to classify any given set of testing sensory data into one of the three health states. This can be accomplished simply by defining a normalized distance, the $z$-score for this example, between the testing data and the sensor output distribution for each health state. The normalized distance measures the divergence of the testing data from the sensor output distribution of each health state, and consequently the testing data should be classified into the health state which has the smallest normalized distance.

To facilitate the diagnostics process, boundaries between two health states will be identified by one neutral point that leads to three equal normalized distances as shown in Figure 2. The natural point $\left(X_{1-2}\right)$ between $H S_{1}$ and $H S_{2}$ can be calculated using Eq. (10), and the natural point $\left(X_{2-3}\right)$ between $H S_{2}$ and $H S_{3}$ can be also calculated accordingly as shown in Eq. (11). 


$$
\begin{aligned}
& \frac{X_{1-2}-1}{0.9}=\frac{3-X_{1-2}}{0.6}, X_{1-2}=2.2000 \\
& \frac{X_{2-3}-3}{0.6}=\frac{6-X_{2-3}}{1.5}, X_{2-3}=3.8571
\end{aligned}
$$

With the identified neutral points between different health states, the detectability $\left(D_{\mathrm{i}}\right)$ can be evaluated as shown in Eqs. (12), (13), and (14), respectively, using the conditional probability defined in Eq. (9).

$$
\begin{aligned}
D_{1} & =P_{11}=\operatorname{Pr}\left(\text { Detected as } H S_{1} \mid \text { System is at } H S_{1}\right) \\
& =\operatorname{Pr}\left(X \leq X_{1-2} \mid X \sim N\left(1,0.9^{2}\right)\right) \\
& =0.9088 \\
D_{2} & =P_{22}=\operatorname{Pr}\left(\text { Detected as } H S_{2} \mid \text { System is at } H S_{2}\right) \\
& =\operatorname{Pr}\left(X_{1-2} \leq X \leq X_{2-3} \mid X \sim N\left(3,0.6^{2}\right)\right) \\
& =0.8322 \\
D_{3} & =P_{33}=\operatorname{Pr}\left(\text { Detected as } H S_{3} \mid \text { System is at } H S_{3}\right) \\
& =\operatorname{Pr}\left(X \geq X_{2-3} \mid X \sim N\left(6,1.5^{2}\right)\right) \\
& =0.9234
\end{aligned}
$$

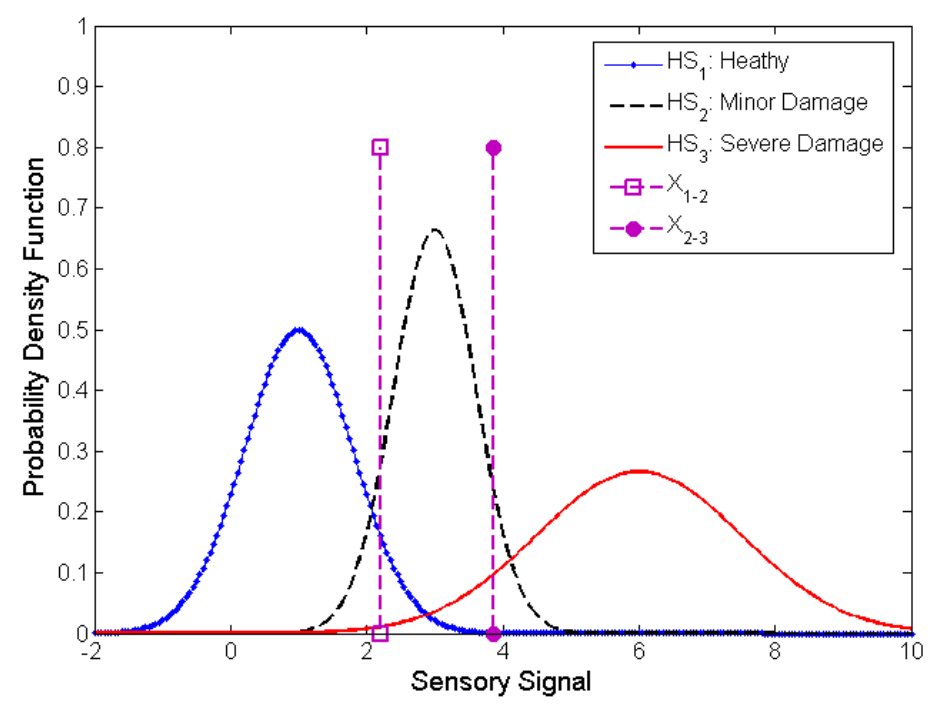

Figure 2: Sensor output distributions neural points

From this mathematical example with analytical evaluation of the detectability, it is clear that statistical distributions and health states classification of sensor outputs are critical for detectability analysis of a given sensing system. Nevertheless, in practical engineering applications, a sensing system will generally contain numerous 
sensors to deal with much more than three health states. Thus, analytical evaluation of sensing system detectability becomes challenging, for example, the calculation of boundaries between different health states. In addition, statistical distributions of sensors' outputs for all health states are not commonly available. To effectively evaluate the detectability for a given sensing system design, a new detectability analysis approach is developed in this study, as detailed in the next subsection, in which the Mahalanobis distance (MD) classifier is employed to handle the uncertainties of sensor output for each system health state and classify sensory data points for detectability calculation.

\subsection{Detectability Analysis Using Mahalanobis Distance Classifier}

This section details a detectability analysis method, which employs Mahalanobis distance (MD) classifiers for the calculation of detectability values based on structural simulation and system health state classification. A MD classifier quantitatively measures the similarity between an online data point and different training data sets from different system health states, and represents the similarity using a Mahalanobis distance measure, whereby a shorter distance indicates a greater similarity. With the MD measure, a testing data point will can be accordingly classified into a health state that induces a minimum Mahalanobis distance value by its training data set. Thus, a MD classifier is capable of categorizing a testing data point measured from an operating system by a sensor network to a predefined system health state from which the sensory data point is most likely measured. Mathematically, the Mahalanobis distance [55] a testing data point from the training data sets of a health state can be calculated by

$$
M D_{i}=\sqrt{\left(X-M_{i}\right)^{T} \Sigma_{i}^{-1}\left(X-M_{i}\right)}
$$

where the $T$ denotes matrix transpose, $\mathrm{X}$ is the given testing data point to be classified, $M_{i}$ and $\Sigma_{i}$ represent the mean vector and the covariance matrix, respectively, for the training data sets for the $i$ th health state, $H S_{i}$,

In the following, one example is used to explain the MD-based health state classification process for detectability analysis. In this example, there are two sensors and three predefined health states $\left(N_{\mathrm{HS}}=3\right)$, in which $\mathrm{HS}_{1}$ is used for system healthy, HS2 for system with minor damage, and HS3 for system with severe damage. The training data information from two different sensors corresponding to different health states is listed in Table 1. In this example, for each health state 100 sensory data points are generated from the distribution information as shown in Table 1, and used as the training data sets. 
Table 1: Predefined System Health States

\begin{tabular}{ccc}
\hline \hline Health State & Sensor 1 & Sensor 2 \\
\hline$H S_{1}$ & $N\left(1.5,0.5^{2}\right)$ & $N\left(1.5,0.5^{2}\right)$ \\
$H S_{2}$ & $N\left(1.5,0.5^{2}\right)$ & $N\left(1.5,0.5^{2}\right)$ \\
$H S_{3}$ & $N\left(1.5,0.5^{2}\right)$ & $N\left(1.5,0.5^{2}\right)$ \\
\hline \hline
\end{tabular}

Table 2: Health States Classification Using MD Classifier

\begin{tabular}{ccccccc}
\hline \hline \multirow{2}{*}{ Testing Data } & & \multicolumn{2}{c}{ Mahalanobis Distance } & Classified \\
\cline { 1 - 2 } \cline { 5 - 6 }$S_{1}$ & $S_{2}$ & & $H S_{1}$ & $H S_{2}$ & $H S_{3}$ & \\
\hline 2.07 & 0.78 & & 4.83 & 73.79 & 27.52 & $H S_{1}$ \\
0.89 & -0.88 & & 5.19 & 48.61 & 2.72 & $H S_{3}$ \\
1.72 & 0.46 & & 1.43 & 62.19 & 17.57 & $H S_{1}$ \\
-2.54 & 0.90 & & 90.97 & 1.13 & 34.76 & $H S_{2}$ \\
0.58 & -1.14 & & 10.12 & 44.93 & 2.13 & $H S_{3}$ \\
\hline \hline
\end{tabular}

First, to demonstrate the MD-based classification process, five testing data points, as shown in the first two columns in Table 2, will be classified to its corresponding health states. Using the MD classifier, the MD values for each testing data set can be calculated with the training data sets using Eq.(15), and a testing data point is classified to a given health state that provides the minimum MD values out of all three. For example, to classify the first testing data point $[2.07,0.78]$ as shown in Table 2 , the MD values are calculated to be $4.83,73.79$, and 27.52 using the training data sets from three different health states, respectively, and consequently this testing data point will be classified as it belongs to $H S_{1}$ since the MD value 4.83 obtained from the first health state is the minimum.

In the following, the PoD matrix for this example will be evaluated and the detectability values for all three predefined health states will be determined by following the same procedure used above. Suppose that there are totally $T_{\mathrm{i}}$ sets (here $T_{\mathrm{i}}=100$ is used) of testing data from the health state $H S_{\mathrm{i}}$, and within which $T_{\mathrm{ij}}$ sets are classified into the health state $H S_{\mathrm{j}}$ by the MD classifier, where $\mathrm{i}, \mathrm{j}=1,2, \ldots, N_{\mathrm{HS}}$, the element $P_{\mathrm{ij}}$ in the PoD matrix can be approximately calculated based on the definition as 


$$
\begin{gathered}
T_{i j}=\left[\begin{array}{ccc}
86 & 0 & 14 \\
0 & 98 & 2 \\
9 & 3 & 88
\end{array}\right] \\
P_{i j} \approx \frac{T_{i j}}{\sum_{j} T_{i j}}=\left[\begin{array}{lll}
0.86 & 0.00 & 0.14 \\
0.00 & 0.98 & 0.02 \\
0.09 & 0.03 & 0.88
\end{array}\right]
\end{gathered}
$$

Since any set of testing data from the health state $H S_{\mathrm{i}}$ will definitely be classified into one of the predefined $N_{\mathrm{HS}}$ health states, the following equation regarding $P_{\mathrm{ij}}$ can be obtained:

$$
\sum_{j=1}^{N_{H S}} P_{i j}=1
$$

The above Eq. (18) suggests that the summation of each row in the PoD matrix will always equal to one. Similarly, the detectability, diagonal terms in the PoD matrix, for the health state $H S_{\mathrm{i}}$ can be obtained as

$$
D_{i}=P_{i i} \approx\left[\begin{array}{lll}
0.86 & 0.98 & 0.88
\end{array}\right]
$$

\section{Reliability Based Robust Design Optimization for Structural Sensing Function Design}

Reliability-based robust design optimization (RBRDO) aims to find the best compromise between cost and reliability by taking uncertainties into account. Moreover, it can be used to ensure the robustness of system designs by minimizing design cost and its uncertainty factors while meeting system reliability or detectability requirements. In this study, the design of a smart sensing system is formulated with RBRDO to optimally allocate PZT sensors so that the robustness of the sensing system performance can be ensured and the detectability requirements for different failure modes can be guaranteed. Generally, the RBRDO formulation of the PZT sensing system design can be presented as a multi objective optimization problem as follows:

$$
\begin{array}{ll}
\text { Min } & \sum_{j=1}^{n f} \mathbf{M}_{j}(\mathbf{x}, \mathbf{d})+\mathbf{Q}_{f}(\mathbf{x}, \mathbf{d}) \\
\text { s.t. } & D_{i}(\mathbf{x}, \mathbf{d}) \geq D_{T}, \quad i=1, \ldots ., n \\
& \mathbf{x}^{L} \leq \mathbf{x} \leq \mathbf{x}^{U}, \quad \mathbf{x} \in R^{n r} \\
& \mathbf{d}^{\mathbf{L}} \leq \mathbf{d} \leq \mathbf{d}^{\mathbf{U}}, \quad \mathbf{d} \in R^{n d}
\end{array}
$$

where the design constraints involved in the sensing systems design framework are detectability requirements considering uncertainties introduced by PZT material properties, manufacturing processes, as well as operating conditions. The design variables are the decision variables for PZT sensors dimension, PZT sensors locations, and 
the parameters for controlling the sensing process. The intent of the sensing systems design optimization is to minimize the cost while ensuring the design robustness and satisfying all detectability requirements. The objective function is thus defined as minimizing PZT sensor size and its variance, where $\mathbf{M}(\mathbf{x}, \mathbf{d})$ is the material and installation cost of PZT sensors for a given sensing system design, $\mathbf{Q}_{f}(\mathbf{x} ; \mathbf{d})$ is robustness penalty cost associated with the quality loss, which is measured by the variability of the cost function $\mathbf{M}, D_{i}$ is the detectability of the sensing system for the $H S_{i}, D_{T}$ is the target detectability, $\mathbf{x}^{U}$ and $\mathbf{x}^{L}$ are upper and lower bounds for the random design variables $\mathbf{x}$, respectively, whereas $\mathbf{d}^{U}$ and $\mathbf{d}^{L}$ are upper and lower bounds for the deterministic design variables $\mathbf{d}$. The parameters $n, n_{r}$, and $n_{f}$, are the numbers of probabilistic constraints, random variables, design variables, and objective functions, respectively.

The RBRDO problem in Eq. (20) contains discrete decision variables for the selection of sensing devices, integer variables for the number of selected sensing devices, as well as continuous variables for the sensor locations. Thus, it is formulated as a mixed-integer nonlinear programming (MINLP) problem [54], and heuristic algorithms such as Genetic Algorithms (GAs) can be used as the optimizer to for the optimization purpose. In this study, the GA is employed for the example problem that will be detailed in the subsequent section. More alternative algorithms for solving the MINLP problem can be found in references [54, 56]. Figure 3 shows the flowchart of the SN design optimization process. As shown in the figure, the process starts from an initial design and goes into the design optimization subroutine (the right hand side grey box), which will carry out the sensing function design cost analysis, call the detectability analysis subroutine (the left hand side grey box) to evaluate the performance of the sensing function at the current design, and execute the optimizer to generate the new designs if the optimality condition is not met. In the detectability analysis subroutine, the detectability analysis as discussed in the previous section will be carried out. Before solving the optimization problem, valid system computer simulation models have to be built and structural simulations have to be accomplished so that the training and testing data sets for each predefined health state are available at the iterative design optimization process for detectability analysis. 


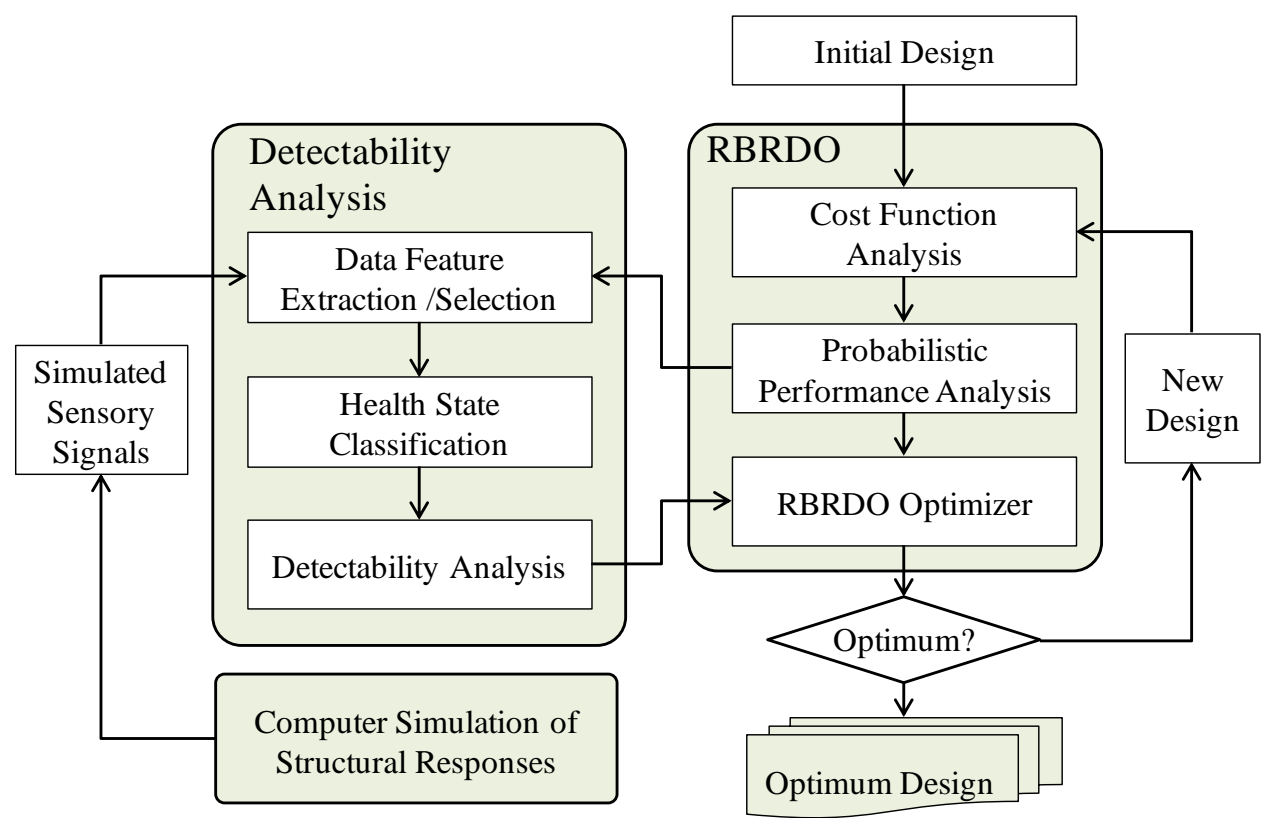

Figure 3: RBRDO Flowchart for Detectability-Based Sensing Function Design

\section{Case Studies}

In this section, the developed reliability-based robust design optimization technique for smart sensing function design using piezoelectric materials is demonstrated two design case studies. In the first case study, the sensing function will be design for a rectangular aircraft wing panel considering rivet joint failures, whereas the second case study designs smart sensing function for power transformer mechanical fault diagnosis. The rest of this section details the two design case studies and results using the developed RBRDO sensing function design methodology.

\subsection{Sensing Function Design for A Rectangular Aircraft Wing Panel}

This subsection demonstrates the proposed RBRDO approach by designing PZT material based sensor patches for a rectangular aircraft wing panel. As shown in Figures 4 and 5, the aircraft wing panel considered in this study has dimensions of two meters by one meter and is fastened by eight rivet joints, as indicated by L1 to L8, respectively. There is a harmonic force $\mathrm{F}$ with a frequency of $120 \mathrm{~Hz}$ applied in the middle of panel to detect joint failures. In this case study, eight health states are defined based on the different rivet joint failure and combinations, as listed in Table 3. To identify the healthy state of the panel, four square PZT sensors are attached to the surface of panel. The electrical potential signal, obtained out of the PZT sensors, is used to identify the health state based on Mahalanobis distance. 


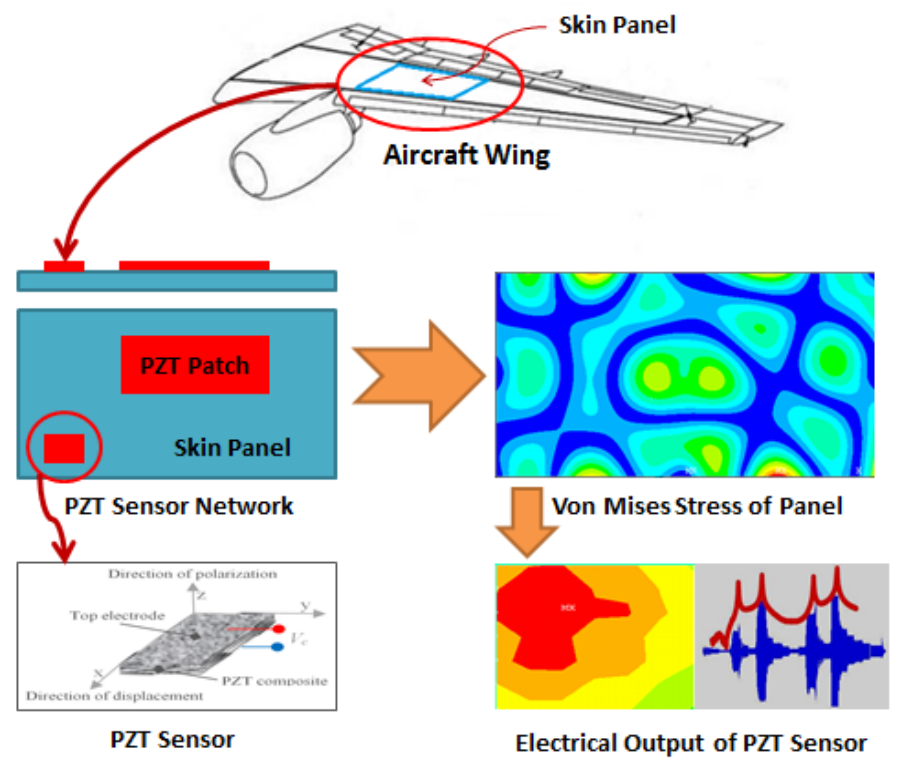

Figure 4: Sensing Function Design of an Aircraft Wing Panel Using Piezoelectric Materials

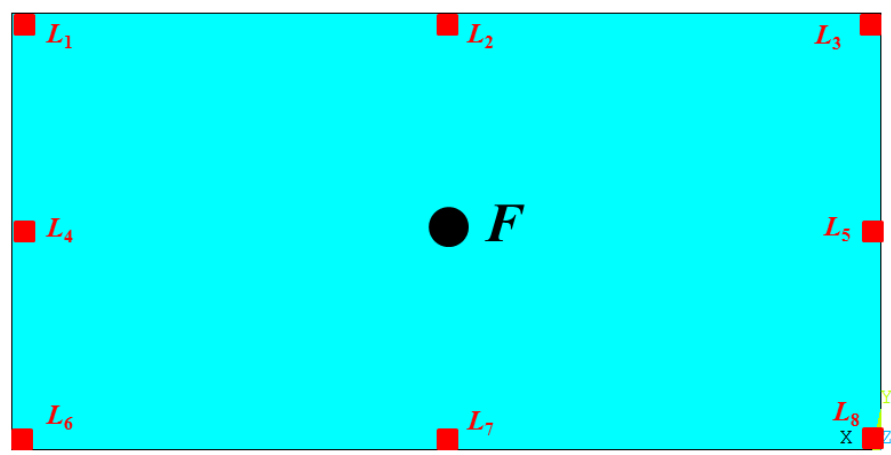

Figure 5: Rectangular Aircraft Wing Panel with Indicated Rivet Joints

Table 3: Health States Definition of Aircraft Wing Panel Case Study

\begin{tabular}{lllllllll}
\hline \hline Health States & $\mathrm{HS}_{0}$ & $\mathrm{HS}_{1}$ & $\mathrm{HS}_{2}$ & $\mathrm{HS}_{3}$ & $\mathrm{HS}_{4}$ & $\mathrm{HS}_{5}$ & $\mathrm{HS}_{6}$ & $\mathrm{HS}_{7}$ \\
\hline Joint Failure & None & L6 & L7 & L4 & L6, L7 & L6, L4 & L4, L7 & L4, L6, L7 \\
\hline \hline
\end{tabular}

The objective function of this RBRDO is to minimize PZT sensor size and its variance while the detectability of each health state meets its requirement to be greater than the target 0.99 . For each sensor, the coordinate and side length of each squared PZT sensor are chosen as design variables, thus totally there are 12 design variables for this case study. All the design variables are assumed to be normal distributed and their upper and lower bounds of means 
and standard deviation are shown in Table 4. Besides the random design variables, three geometric random parameters are also considered in this case study, as listed in Table 5.

Table 4: Design Variables of the Rectangular Aircraft Wing Panel Case Study

\begin{tabular}{llllll}
\hline \hline Variables & Definition & LB & UB & Distribution & SD \\
\hline $\mathrm{X}_{1}$ & X coordinate of the first sensor & 0 & 2 & Normal & 0.02 \\
$\mathrm{Y}_{1}$ & Y coordinate of the first sensor & 0 & 1 & Normal & 0.02 \\
$\mathrm{~S}_{1}$ & Side length of the first sensor & 0 & 0.1 & Normal & $2 \mathrm{e}-3$ \\
$\mathrm{X}_{2}$ & $\mathrm{X}$ coordinate of the second sensor & 0 & 2 & Normal & 0.02 \\
$\mathrm{Y}_{2}$ & Y coordinate of the second sensor & 0 & 1 & Normal & 0.02 \\
$\mathrm{~S}_{2}$ & Side length of the second sensor & 0 & 0.1 & Normal & $2 \mathrm{e}--3$ \\
$\mathrm{X}_{3}$ & $\mathrm{X}$ coordinate of the third sensor & 0 & 2 & Normal & 0.02 \\
$\mathrm{Y}_{3}$ & Y coordinate of the third sensor & 0 & 1 & Normal & 0.02 \\
$\mathrm{~S}_{3}$ & Side length of the third sensor & 0 & 0.1 & Normal & $2 \mathrm{e}-3$ \\
$\mathrm{X}_{4}$ & $\mathrm{X}$ coordinate of the fourth sensor & 0 & 2 & Normal & 0.02 \\
$\mathrm{Y}_{4}$ & Y coordinate of the fourth sensor & 0 & 1 & Normal & 0.02 \\
$\mathrm{~S}_{4}$ & Side length of the fourth sensor & 0 & 0.1 & Normal & $2 \mathrm{e}-3$ \\
\hline \hline
\end{tabular}

Table 5: Random Parameters of the Rectangular Aircraft Wing Panel Case Study

\begin{tabular}{ccccc}
\hline \hline Parameters & Definition & Distribution & Mean & SD \\
\hline L & Length of Panel & Normal & 10 & 0.1 \\
W & Width of Panel & Normal & 5 & 0.05 \\
F & Amplitude of Force & Normal & 1000 & 50 \\
\hline \hline
\end{tabular}

In this case study, RBRDO of sensing system for the rectangular panel can be formulated as follows: 


$$
\begin{aligned}
\text { Minimize } & \sum_{j=1}^{4} S_{j}+\operatorname{Var}\left(\sum_{j=1}^{4} S_{j}\right) \\
\text { subject to }: & D_{i}(X, Y, S) \geq D_{T}, \quad i=0, \ldots, 7 \\
& x^{L} \leq x \leq x^{U}, \quad X \in R^{n r} \\
& y^{L} \leq y \leq y^{U}, \quad Y \in R^{n r} \\
& s^{L} \leq s \leq s^{U}, \quad S \in R^{n r}
\end{aligned}
$$

where the objective function is to minimize the total area of PZT sensors and its variance, as denoted by the summation of the area $S_{\mathrm{j}}$ for each sensor unit. The detectability target is set as 0.99 for all seven health states. To obtain the electrical potential response of PZT attached on the panel, a 3D finite element model is established in ANSYS 12. Figure 6 shows a particular sensor layout and its vibration displacement of health states $H S_{0}$. The electrical potential contours of four PZT sensors are shown in Figure 7.

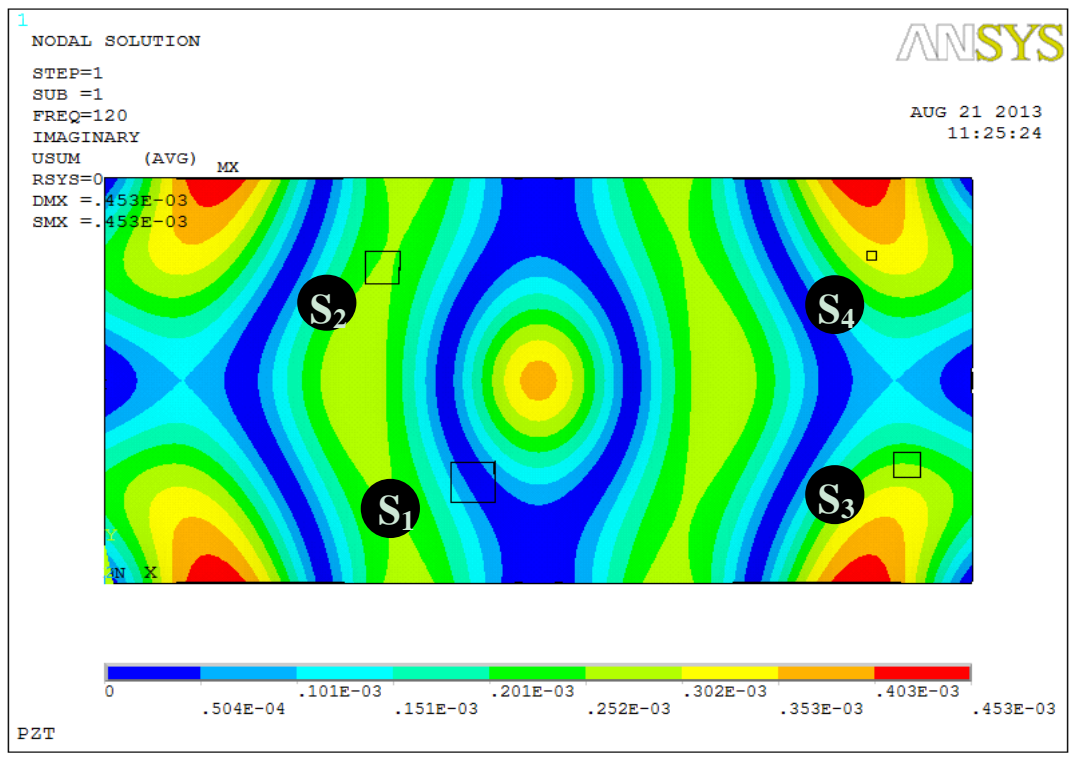

Figure 6: Sensor Layout and Vibration Amplitude Contour of the Panel for $\boldsymbol{H} S_{0}$ 


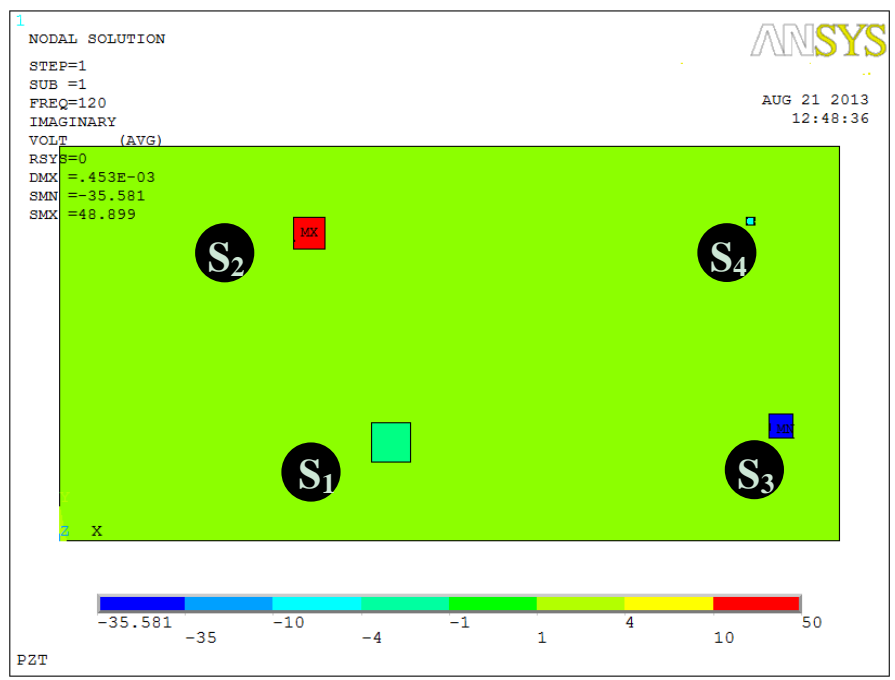

Figure 7: Electrical Potential Contours of Four PZT Sensors

The location and size of PZT sensor can directly determine the electrical potential response given a certain scenario. In this case, sensor $S_{2}$ and $S_{3}$ get relative significant electrical response while there is almost no obvious electrical potential for sensor $S_{1}$ and $S_{4}$. Due to the uncertainties involved from not only the whole structural but also the sensing system, the signal output of each sensor is also a random value. Generic algorithm is used to search optimal solution for the RBRDO problem, and the optimal design is obtained after 15 generations. The design history is shown in Table 6 while the corresponding detectability and cost are shown in Table 7 in which $\mathrm{C}_{\mathrm{T}}$ represents the total cost comprising design cost $M$ and quality lost $Q_{c}$. The corresponding detectability of each generation is also shown in Figure 8. Figure 9 demonstrates the cost distribution of initial and optimum design while Figure 10 shows the optimum layout of sensor system. As shown in the Figure 9; both the mean and variance of design cost are increased slightly while the detectability of sensing system is improved significantly. 
Table 6: Iterative Design History of RBRDO for the Aircraft Wing Panel Case Study

\begin{tabular}{ccccccccccccc}
\hline Iter. & $\mathrm{X} 1$ & $\mathrm{Y} 1$ & $\mathrm{~S} 1$ & $\mathrm{X} 2$ & $\mathrm{Y} 2$ & $\mathrm{~S} 2$ & $\mathrm{X} 3$ & $\mathrm{Y} 3$ & $\mathrm{~S} 3$ & $\mathrm{X} 4$ & $\mathrm{Y} 4$ & $\mathrm{~S} 4$ \\
\hline 1 & 12.00 & 12.00 & 3.00 & 110.00 & 60.00 & 3.00 & 12.00 & 60.00 & 3.00 & 110.00 & 12.00 & 3.00 \\
2 & 23.64 & 17.87 & 3.80 & 175.64 & 71.10 & 3.20 & 18.52 & 65.55 & 6.79 & 175.70 & 61.45 & 5.77 \\
3 & 71.91 & 27.42 & 5.11 & 115.07 & 71.65 & 3.44 & 68.83 & 64.02 & 6.07 & 139.60 & 67.59 & 3.03 \\
4 & 71.91 & 25.34 & 3.45 & 115.07 & 77.63 & 3.44 & 68.83 & 64.02 & 6.07 & 168.58 & 69.27 & 3.03 \\
5 & 71.91 & 25.34 & 3.45 & 115.07 & 77.63 & 3.44 & 68.83 & 64.02 & 6.07 & 139.60 & 69.27 & 3.03 \\
6 & 73.91 & 25.34 & 3.45 & 115.07 & 77.63 & 3.44 & 68.83 & 64.02 & 4.07 & 141.60 & 69.27 & 3.03 \\
7 & 75.91 & 25.34 & 3.45 & 115.07 & 77.63 & 3.44 & 68.83 & 64.02 & 4.07 & 141.60 & 69.27 & 3.03 \\
8 & 75.91 & 25.34 & 3.45 & 167.15 & 77.63 & 3.00 & 68.83 & 60.31 & 4.07 & 141.60 & 69.27 & 3.03 \\
9 & 75.91 & 25.34 & 3.45 & 167.15 & 77.63 & 3.00 & 68.83 & 60.31 & 4.07 & 141.60 & 69.27 & 3.03 \\
10 & 73.91 & 25.34 & 3.45 & 115.07 & 77.63 & 3.00 & 68.83 & 60.31 & 4.07 & 141.60 & 69.27 & 3.03 \\
11 & 73.91 & 25.34 & 3.45 & 115.07 & 77.63 & 3.00 & 68.83 & 60.31 & 4.07 & 142.60 & 69.27 & 3.03 \\
12 & 76.91 & 25.34 & 3.45 & 115.07 & 79.35 & 3.00 & 68.83 & 60.31 & 3.07 & 142.60 & 69.27 & 3.03 \\
13 & 76.91 & 25.34 & 3.45 & 115.07 & 79.35 & 3.00 & 68.83 & 60.31 & 3.07 & 142.60 & 69.27 & 3.03 \\
14 & 76.91 & 25.34 & 3.45 & 115.07 & 79.35 & 3.00 & 68.83 & 60.31 & 3.07 & 142.60 & 69.27 & 3.03 \\
15 & 76.91 & 25.34 & 3.45 & 115.07 & 79.35 & 3.00 & 68.83 & 60.31 & 3.07 & 142.60 & 69.27 & 3.03 \\
\hline \hline
\end{tabular}


Table 7: Detectability and Cost of RBRDO for the Aircraft Wing Panel Case Study

\begin{tabular}{cccccccccccc}
\hline \hline Iter. & D0 & D1 & D2 & D3 & D4 & D5 & D6 & D7 & M & Qc & CT \\
\hline 1 & 0.79 & 0.96 & 0.93 & 0.95 & 0.90 & 0.97 & 0.94 & 0.95 & 36.00 & 5.76 & 41.76 \\
2 & 0.96 & 1.00 & 0.98 & 0.98 & 1.00 & 1.00 & 0.99 & 1.00 & 104.0 & 16.70 & 120.7 \\
3 & 0.97 & 0.99 & 0.97 & 0.99 & 1.00 & 1.00 & 1.00 & 0.99 & 84.00 & 13.45 & 97.45 \\
4 & 0.98 & 0.98 & 1.00 & 0.99 & 0.99 & 1.00 & 1.00 & 0.98 & 69.76 & 11.10 & 80.86 \\
5 & 0.99 & 1.00 & 1.00 & 1.00 & 1.00 & 1.00 & 1.00 & 0.99 & 69.76 & 11.21 & 80.97 \\
6 & 0.99 & 1.00 & 1.00 & 1.00 & 1.00 & 1.00 & 1.00 & 0.99 & 49.48 & 7.91 & 57.39 \\
7 & 0.99 & 1.00 & 1.00 & 1.00 & 1.00 & 1.00 & 1.00 & 0.99 & 49.48 & 7.93 & 57.41 \\
8 & 0.98 & 0.99 & 1.00 & 0.99 & 1.00 & 1.00 & 1.00 & 1.00 & 46.62 & 7.47 & 54.09 \\
9 & 0.98 & 0.99 & 1.00 & 0.99 & 1.00 & 1.00 & 1.00 & 1.00 & 46.62 & 7.49 & 54.11 \\
10 & 0.98 & 0.99 & 1.00 & 0.99 & 1.00 & 1.00 & 1.00 & 0.99 & 46.62 & 7.44 & 54.06 \\
11 & 0.98 & 0.99 & 1.00 & 0.99 & 1.00 & 1.00 & 1.00 & 0.99 & 46.62 & 7.50 & 54.12 \\
12 & 0.99 & 0.99 & 1.00 & 1.00 & 1.00 & 0.99 & 1.00 & 1.00 & 39.48 & 6.35 & 45.83 \\
13 & 0.99 & 0.99 & 1.00 & 1.00 & 1.00 & 0.99 & 1.00 & 1.00 & 39.48 & 6.31 & 45.79 \\
14 & 0.99 & 0.99 & 1.00 & 1.00 & 1.00 & 0.99 & 1.00 & 1.00 & 39.48 & 6.29 & 45.77 \\
15 & 0.99 & 0.99 & 1.00 & 1.00 & 1.00 & 0.99 & 1.00 & 1.00 & 39.48 & 6.33 & 45.81 \\
\hline \hline
\end{tabular}

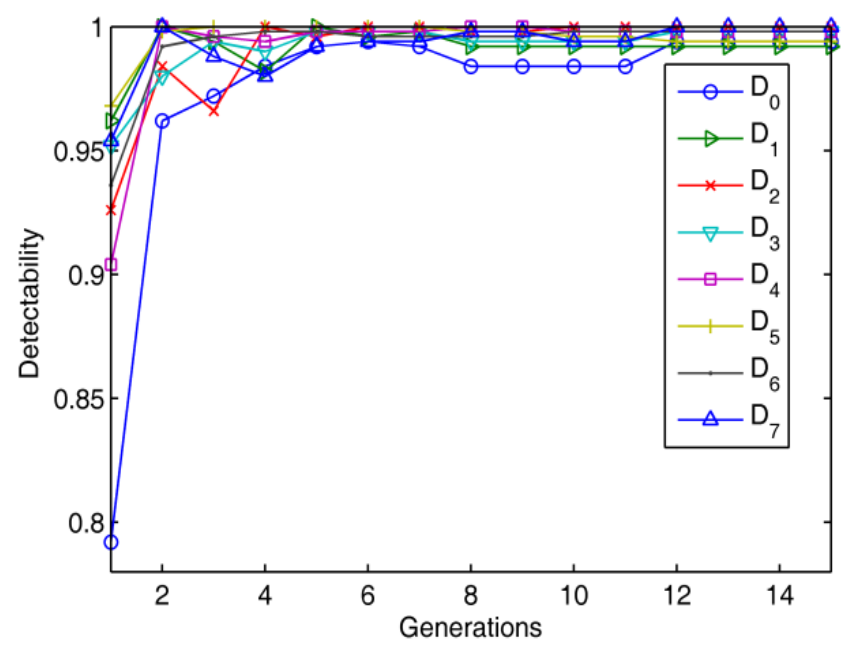

Figure 8: The Detectability History of RBRDO for the Aircraft Wing Panel Case Study 


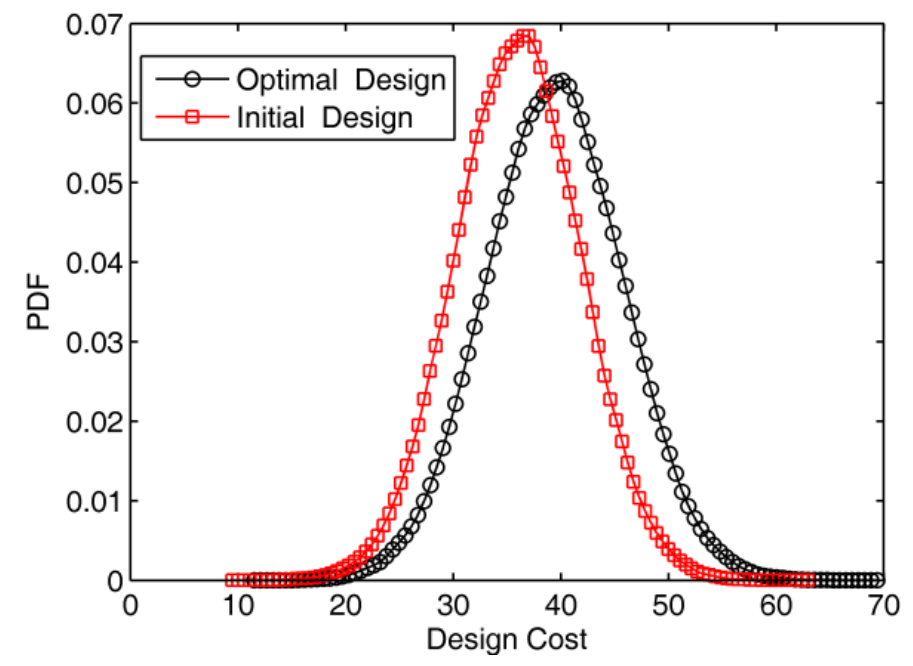

Figure 9: The Cost Distributions of Initial and Optimal Design

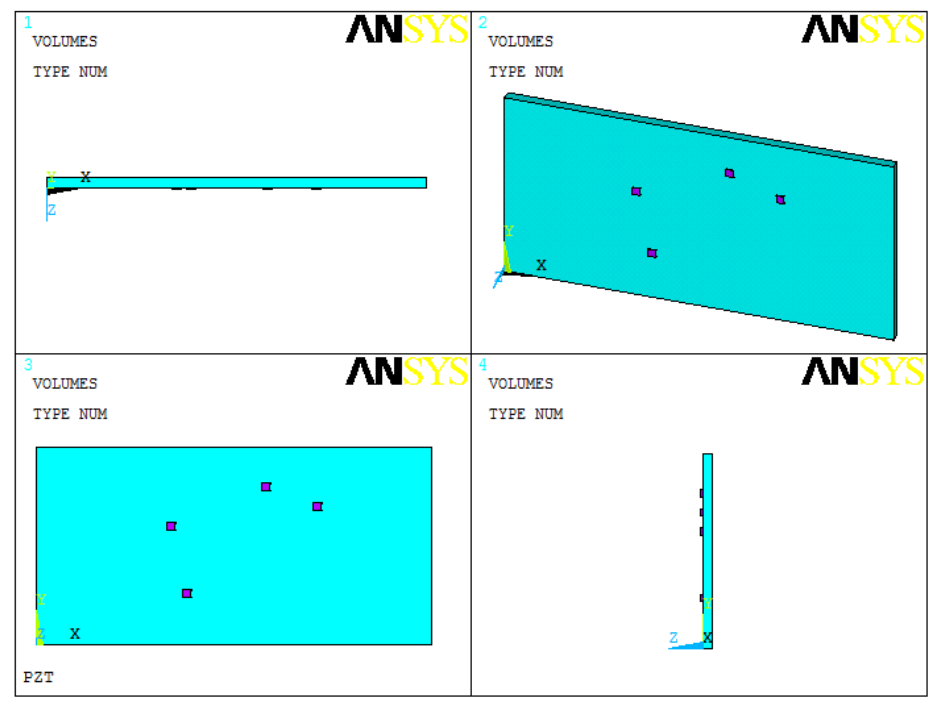

Figure 10: Layout of the Sensors for the Optimum Design

\subsection{Sensing Function Design for Power Transformer Mechanical Fault Diagnostics}

Power transformers are among the most expensive elements of high-voltage power systems. The monitoring of power transformers enables the transition from the traditional time-based maintenance to the condition-based maintenance, resulting in significant reductions in maintenance costs. Due to the difficulties of direct measurement inside the transformer, the data that are actually most often used for both diagnosis and prognosis of transformers are obtained through indirect measurements. For example, measurements of temperature are firstly accomplished at 
accessible points and a modeling of the gradient can then be used to induce the maximum temperature in some areas; electric parameters and analysis of moisture content of the cooling oil are often performed for the diagnosis and condition-based maintenance of transformers, with frequency response analysis of electric characteristics being common; the vibrations of the magnetic core and of the windings could characterize transitory overloads and permanent failures before any irreparable damage occurs. This case study aims at designing an optimum PZT sensor system on the front wall surface of a power transformer. The measurements of the transformer vibration responses induced by the magnetic field loading enables the detection of mechanical failures of winding support joints inside the transformer.

In this study, the winding support joint loosening is considered as the failure mode, the detection of which will be realized by collecting the vibration signal, induced by the magnetic field loading with a fixed frequency on the power transformer core, using the optimally designed PZT sensor system at the external surface of the transformer. The validated finite element (FE) model of a power transformer was created in ANSYS as shown in Figure11, where one exterior wall is concealed to make the interior structure visible. Figure 12 shows 12 simplified winding support joints with 4 for each winding. The transformer is fixed at the bottom surface and a vibration load with the frequency of $120 \mathrm{~Hz}$ is applied to the transformer core. The joint loosening was realized by reducing the stiffness of the joint itself. Different combinations of the loosening joints will be treated as different health states of the power transformer which will be detailed in the next subsection. The uncertainties in this case study are modeled as random parameters with corresponding statistical distributions listed in Table 8. These uncertainties will be propagated into the structural vibration responses and will be accounted for when designing an optimum PZT sensor system.

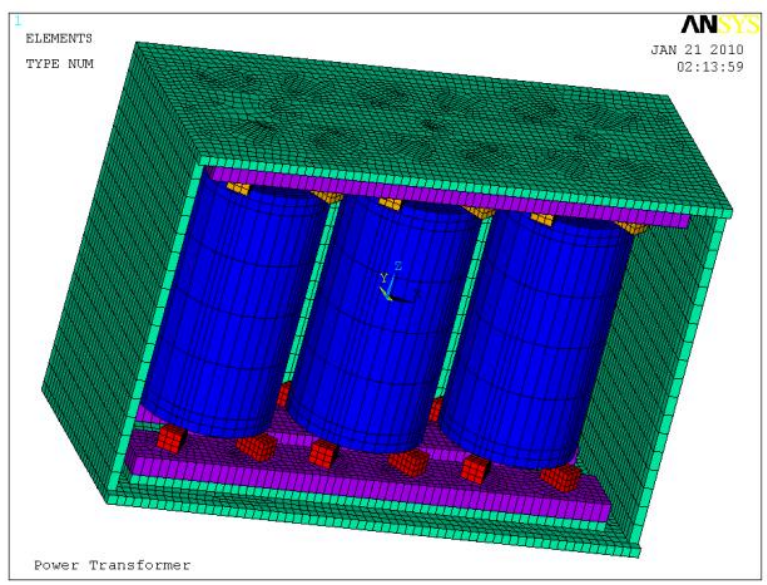


Figure 11: A Power Transformer FE Model (without the covering wall)

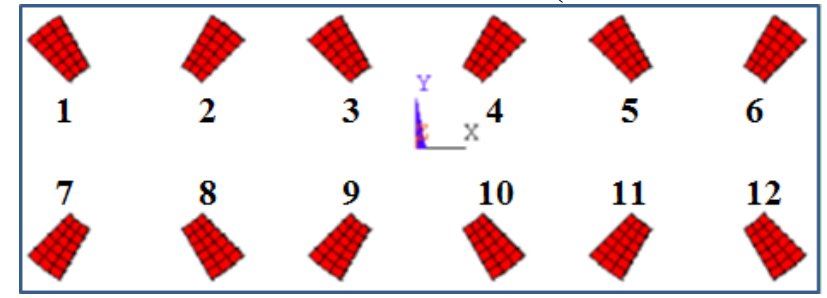

Figure 12: Winding Support Joints and Their Numberings

Table 8: Random Property of the Power Transformer

\begin{tabular}{ll}
\hline \hline Random Variable & Randomness (cm,g,degree) \\
\hline Young's modulus of support joint & $N\left(2 \mathrm{e} 12,4 \mathrm{e} 10^{2}\right)$ \\
Young's modulus of loosening joints & $N\left(2 \mathrm{e} 10,4 \mathrm{e} 8^{2}\right)$ \\
Young's modulus of winding & $N\left(1.28 \mathrm{e} 12,3 \mathrm{e} 10^{2}\right)$ \\
Poisson ratio of joints & $N\left(0.27,0.0054^{2}\right)$ \\
Poisson ratio of winding & $N\left(0.34,0.0068^{2}\right)$ \\
Density of joints & $N\left(7.85,0.157^{2}\right)$ \\
Density of windings & $N\left(8.96,0.179^{2}\right)$ \\
Length of PZT layer & $N\left(\mathrm{~S},(0.05 * \mathrm{~S})^{2}\right)$ \\
\hline
\end{tabular}

For the purpose of demonstrating the proposed design methodology, 9 representative health states shown in Table 9 were selected from all possible combinations of 12 winding support joint failures. Among these 9 selected health states, $H S 1$ denotes the healthy condition without any loosening joint, whereas $H S 2$ to $H S 9$ are health states with either one or two loosening joints. According to the statistical properties of random parameters in Table 8, 200 sets of random samples were generated and the simulations for each of 9 health states were carried out and the average electoral potentials of PZT layers are extracted as sensor signals and were saved as the simulation results.

Table 9: Definition of System Health States

\begin{tabular}{cccccccccc}
\hline \hline Health State & 1 & 2 & 3 & 4 & 5 & 6 & 7 & 8 & 9 \\
\hline Loosening Joints & - & 1 & 2 & 3 & 1,2 & 1,3 & 1,5 & 1,9 & 1,11 \\
\hline \hline
\end{tabular}


The displacement contour of the healthy state power transformer at the nominal values of the random parameters from the structural simulation is shown in Figure 13.

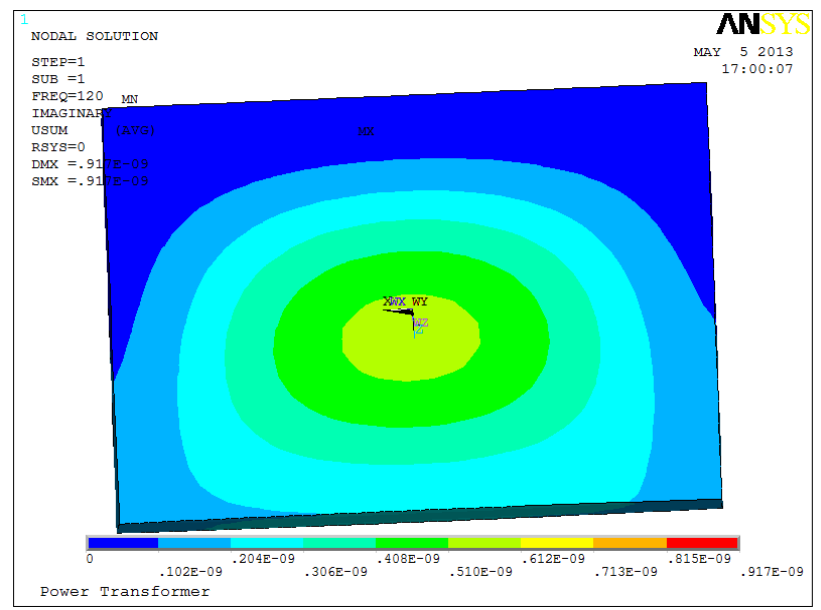

Figure 13: Vibration Contour of the Winding Support for the Healthy State of Power Transformer

Similar to the first case study, applying the developed sensor network design methodology for the power transformer case study, the design problem can be formulated as follows:

$$
\begin{aligned}
\text { Minimize } & \sum_{j=1}^{4} S_{j}+\operatorname{Var}\left(\sum_{j=1}^{4} S_{j}\right) \\
\text { subject to }: & D_{i}(X, Y, S) \geq D_{T}, \quad i=1, \ldots ., 9 \\
& x^{L} \leq x \leq x^{U}, \quad X \in R^{n r} \\
& y^{L} \leq y \leq y^{U}, \quad Y \in R^{n r} \\
& s^{L} \leq s \leq s^{U}, \quad S \in R^{n r}
\end{aligned}
$$

where the objective function is to minimize the total area of PZT sensors and its variance, and the detectability target has been set to 0.90 for all nine health states. To conduct the design optimization of the piezoelectric material based sensor network, the simulation has been conducted with random inputs as given in Table 8 . The first 100 sets of simulation results were used as the training data set and the others were used as testing data set. These simulation results were later used to evaluate the detectability of sensor system. As mentioned in the previous section, this case study problem is formulated as designing a PZT sensor system on the surface of the covering wall of the power transformer to minimize the cost of the SN while satisfying the detectability constraints for each health state, i.e., the 
detectability should be greater than a target detectability of 0.9 . In the RBRDO framework, the cost function is defined as the sum of two parts: the mean and variance of total PZT area; the total number of PZT sensor are three and the PZT sensors are assumed to be square. The design variables in this case study include: (1) location of each PZT sensor, (2) length of each PZT sensor. The RBRDO problem in this case study was solved using the genetic algorithm. With the target detectability being 0.9 , we obtained the optimum sensor system design on the outer wall surface $(140 \mathrm{~cm} \times 90 \mathrm{~cm})$ with totally 4 sensors, as shown in Table 10 . Figures 14 and 15 show the detectability and cost history of RBRDO process for 9 health states, respectively, whereas Figure 16 demonstrates the distribution of initial and optimum sensor system designs. The final optimum sensor system is illustrated in Figure 17.

Table 10: Design History during RBRDO

\begin{tabular}{ccccccccccccc}
\hline \hline Iter. & $\mathrm{X} 1$ & $\mathrm{Y} 1$ & $\mathrm{~S} 1$ & $\mathrm{X} 2$ & $\mathrm{Y} 2$ & $\mathrm{~S} 2$ & $\mathrm{X} 3$ & $\mathrm{Y} 3$ & $\mathrm{~S} 3$ & $\mathrm{X} 4$ & $\mathrm{Y} 4$ & $\mathrm{~S} 4$ \\
\hline 1 & -40.00 & -35.00 & 8.00 & -40.00 & 20.00 & 8.00 & 40.00 & -35.00 & 8.00 & 40.00 & 45.00 & 8.00 \\
2 & -36.87 & -15.35 & 4.93 & -32.03 & 0.22 & 1.50 & 0.80 & -23.61 & 5.71 & 0.67 & 0.84 & 7.68 \\
3 & -40.00 & -35.00 & 8.00 & -21.56 & 0.26 & 8.00 & 40.00 & -35.00 & 8.86 & 40.00 & 0.25 & 7.22 \\
4 & -14.87 & -22.73 & 7.66 & -56.55 & 20.00 & 8.00 & 0.41 & -35.00 & 8.00 & 0.91 & 0.22 & 7.16 \\
5 & -46.05 & -35.00 & 1.82 & -40.00 & 0.83 & 6.37 & 0.44 & -35.00 & 6.60 & 0.95 & 0.05 & 6.17 \\
6 & -52.32 & -34.00 & 1.82 & -12.50 & 0.13 & 9.00 & 40.00 & -35.00 & 6.30 & 0.95 & 0.16 & 8.91 \\
7 & -71.74 & -35.00 & 8.00 & -40.00 & 0.13 & 3.96 & 0.14 & -54.12 & 2.19 & 40.00 & 0.27 & 2.88 \\
8 & -46.05 & -35.00 & 3.19 & -40.00 & 0.26 & 7.81 & 40.00 & -35.00 & 2.19 & 40.00 & 0.27 & 1.88 \\
9 & -46.05 & -35.00 & 3.19 & -40.00 & 0.26 & 3.82 & 40.00 & -43.45 & 2.19 & 40.00 & 0.27 & 2.88 \\
10 & -71.74 & -35.00 & 2.51 & -40.00 & 0.13 & 3.82 & 40.00 & -43.45 & 2.19 & 40.00 & 0.96 & 2.88 \\
\hline \hline
\end{tabular}




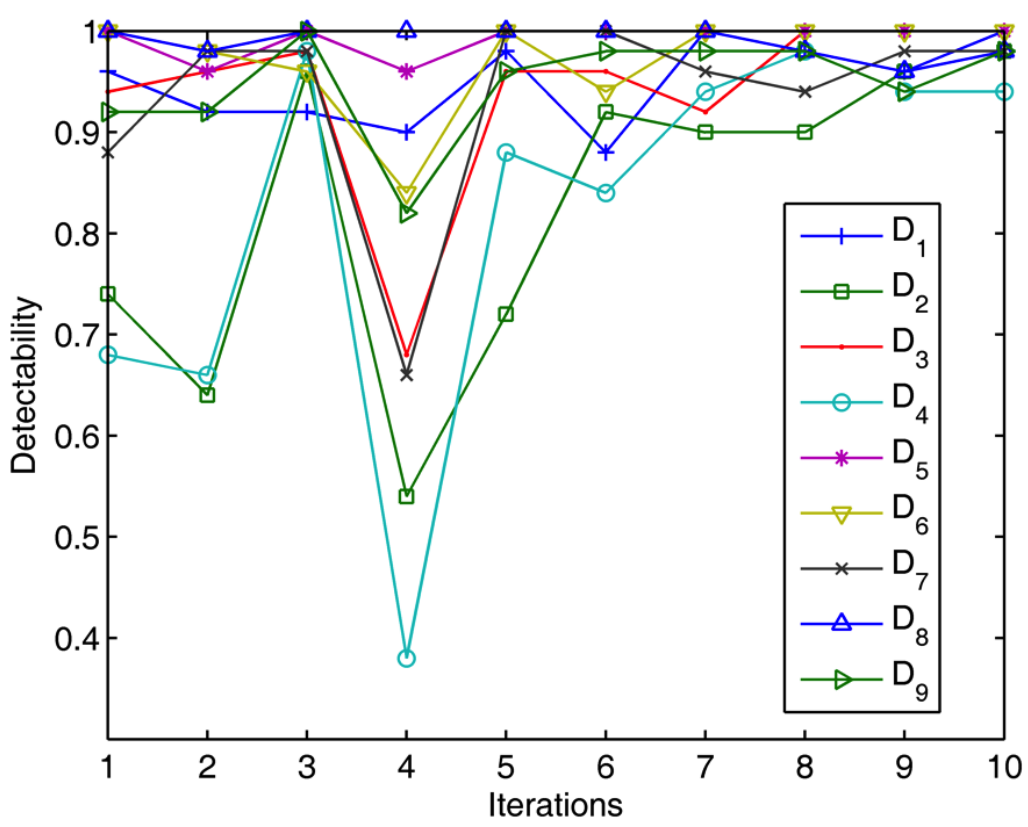

Figure 14: Detectability History during RBRDO

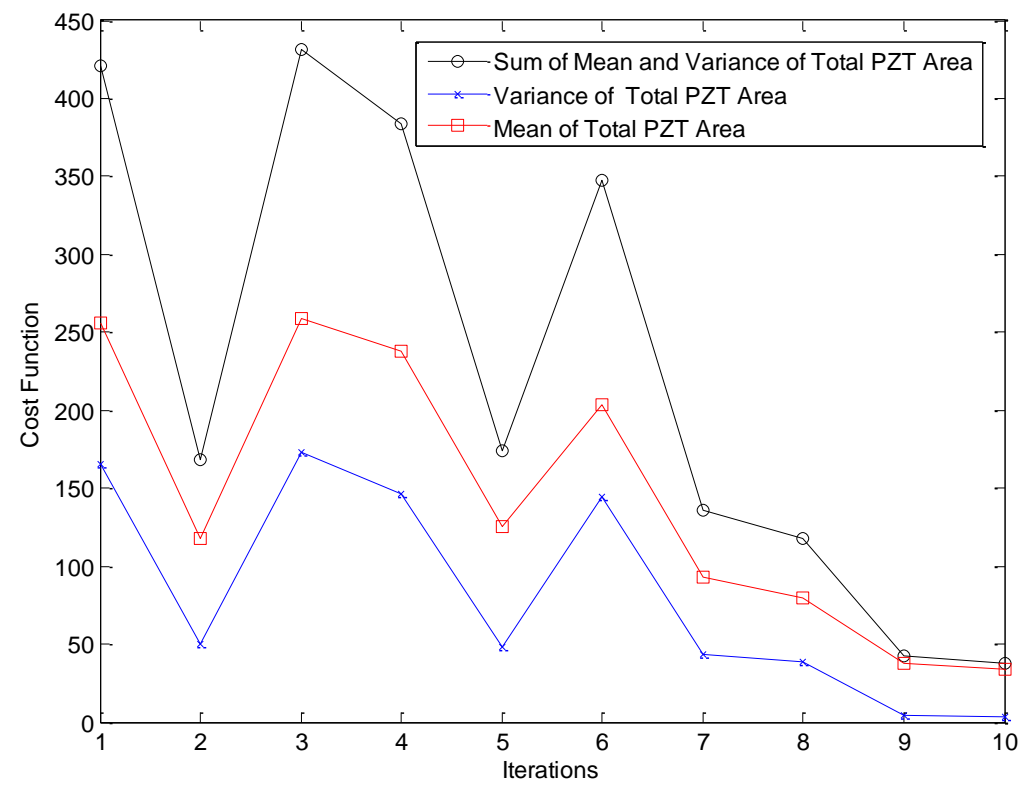

Figure 15: Cost History during RBRDO 


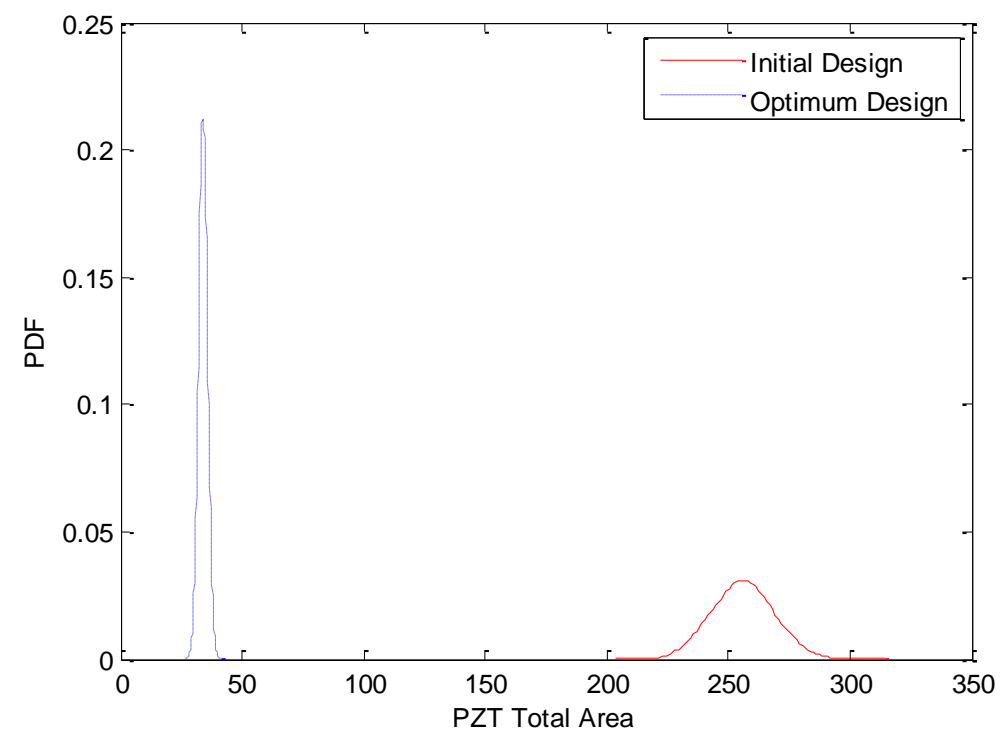

Figure 16: Cost Distribution of Initial and Optimum Designs

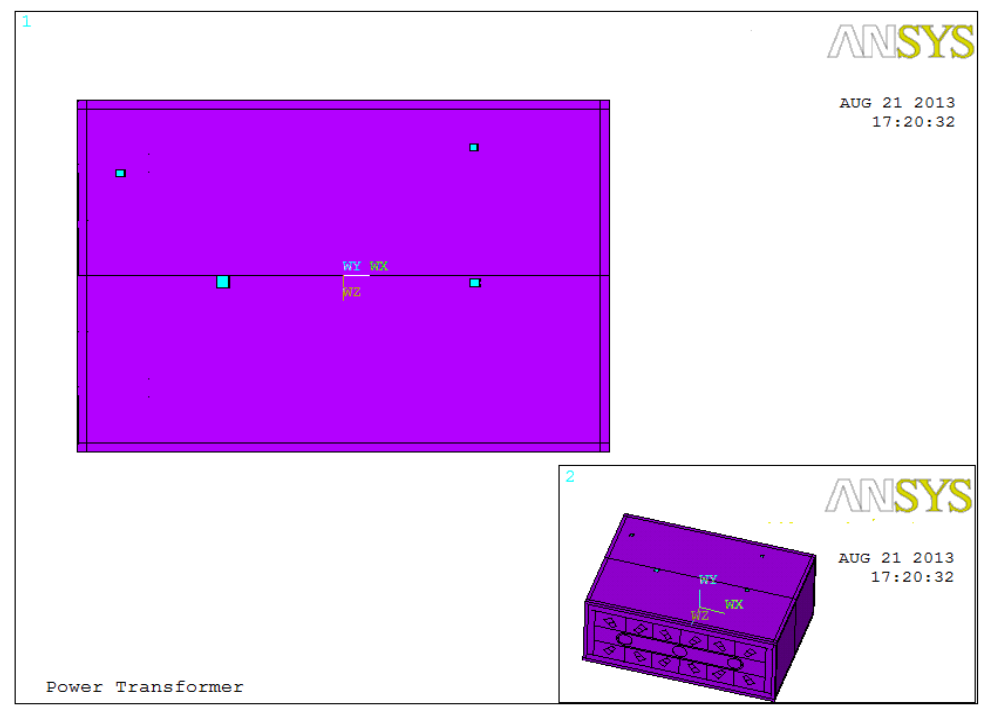

Figure 17: Optimal Layout of Sensor System

\subsection{Remarks on the Case Studies}

This subsection provides the remarks of the two case studies as presented in the previous two subsections.

Remark 1. In this study, the detectability of the designed sensor networks using piezoelectric materials has been calculated based on finite element simulation models. Note that the simulation model is an important factor that would affect the results of sensor network designs. In the previous study, the experimental validation for the 
structure simulation models coupled with piezoelectric materials has been performed and reported in one of the authors' previous publications [57], where an excellent agreement of open circuit voltage between the simulation and the test has been reported. In the studies presented in this paper, same modeling and simulation strategies as reported in [57] have been used. As this study is focused on developing a new probabilistic design framework based on the new detectability concept for generic smart sensing systems, the authors assumed that the simulation models used in both case studies are valid.

Remark 2. In the power transformer case study, the electrical field inside the power transformer could have an impact on the piezoelectric sensors attached to it. However, it is assumed that the impact of electric field inside the power transformer windings on piezoelectric sensor outputs is minor compared to the one contributed by power transformer vibrations. Thus, to maintain the case study in a certain level of simplicity without losing the generosity to demonstrate the proposed sensor network design methodology, the internal electric field impact has been neglected in this study.

\section{Conclusion}

This paper presented a reliability-based robust design optimization approach for the development of piezoelectric materials based structural sensing systems for failure diagnostics and prognostics, to ensure the robustness of sensing performance. In the proposed approach, a detectability measure is defined to evaluate the performance of any given sensing system, and the sensing system design problem can be formulated to maximize detectability for different failure modes by optimally allocating piezoelectric materials into a target structure. This formulation can be conveniently solved within a reliability-based robust design framework to ensure design robustness while considering the uncertainties. Two case studies are employed to demonstrate the effectiveness of the proposed methodology in developing multifunctional material sensing systems. Two case studies were used to demonstrate the developed RBRDO approach with a quantitative detectability measure for piezoelectric material based structural sensing system design. The case study results indicated that the developed approach is very effective for the structural sensing design problems considering the uncertainties, in that it takes into account the performance variability in the design optimization process thus the design cost can be reduced while simultaneously the performance robustness can be ensured and all detectability requirements can be satisfied. 


\section{Acknowledgement}

This research is partially supported by National Science Foundation through Faculty Early Career Development (CAREER) award (CMMI- 1351414) and the Award (CMMI-1200597), and Department of Transportation the University Transportation Center (UTC) program through Midwest Transportation Center (MTC).

\section{Reference}

[1] Jardine, A. K., Lin, D., \& Banjevic, D.. A review on machinery diagnostics and prognostics implementing condition-based maintenance. Mechanical systems and signal processing, 20(7), 2006, 1483-1510.

[2] Dekker, R., “Applications of maintenance optimization models: a review and analysis,” Reliability Engineering System Safety, Vol. 51, 1996, pp. 229-240.

[3] De Oliveira, R., Ramos, C. A., \& Marques, A. T. Health monitoring of composite structures by embedded FBG and interferometric Fabry-Pérot sensors. Computers \& structures, 86(3), 2008 340-346.

[4] Coit, D. W., and Jin, T., "Gamma distribution parameter estimation for field reliability data with missing failure times," IIE Transactions, Vol. 32, No. 12, 2000, pp. 1161-1166.

[5] Elsayed, E. A., "Perspectives and challenges for research in quality and reliability engineering," International Journal of Production Research, Vol. 38, No. 9, 2000, pp. 1953-1976.

[6] Alguindigue, I. E., Loskiewicz-Buczak, A., and Uhrig, R. E., "Monitoring and diagnosis of rolling element bearings using artificial neural networks,"IEEE Transactions Industrial Electronics, Vol. 40, No. 2, 1993, pp. 209-217.

[7] Li, Y., Billington, S., and Zhang, C., "Dynamic prognostic prediction of defect propagation on rolling element bearings," Lubrication Engineering, Vol. 42, No. 2, 1999, pp. 385-392.

[8] Huang, R., Xi, L., Li,X., Richard Liu, C., Qiu, H., and Lee, J., "Residual Life Predictions for Ball Bearings Based on Self-Organizing Map and Back Propagation Neural Network Methods," Mechanical Systems and Signal Processing, Vol. 21, 2007, pp. 193-207.

[9] Zhang, L.,"Bearing fault diagnosis using multi-scale entropy and adaptive neuro-fuzzy inference," Expert Systems With Applications, Vol. 37, No. 8, 2010, pp. 6077-6085.

[10] Martin, K. F.,"Review by discussion of condition monitoring and fault diagnosis in machine tools," International Journal of Machine Tools Manufacturing, Vol. 34, No. 4, 1994, pp. 527-551.

[11] Macian, V., Tormos, B., Olmeda, P., and Montoro, L., "Analytical approach to wear rate determination for internal combustion engine condition monitoring based on oil analysis," Tribology International, Vol. 36, No. 10, 2003, pp. $771-776$. 
[12] Booth, C., and McDonald, J. R., "The use of artificial neural networks for condition monitoring of electrical power transformers," Neuro-Computing, Vol. 23, 1998, pp. 97-109.

[13] Zhao, X., Gao, H., Zhang, G., Ayhan, B., Yan, F., Kwan, C., and Rose, J. L., “Active Health Monitoring of an Aircraft Wing with Embedded Piezoelectric Sensor/Actuator Network: I. Defect Detection, Localization and Growth Monitoring,” Smart Materials and Structures, Vol. 16, 2007, pp. 1208.

[14] Breikin, T., Kulikov, G., Arkov, V., and Fleming, P., "Dynamic Modelling for Condition Monitoring of Gas Turbines: Genetic Algorithms Approach," in 16th IFAC World Congress, 2005.

[15] Wang, P., Youn, B. D., and Hu, C., "A Probabilistic Detectability-Based Sensor Network Design Method for Health Monitoring and Prognostics," Journal of Intelligent Material Systems and Structures, doi: 10.1177/1045389X14541496, 2014.

[16] Zhang, B., and Srihari, S. N., "Fast k-Nearest Neighbor Classification Using Cluster-Based Trees," IEEE Transactions Pattern Analysis and Machine Intelligence, Vol. 26, No. 4, 2004, pp. 525-528.

[17] Alsabti, K., Ranka, S., and Singh, V., “An Efficient k-Means Clustering Algorithm,” Electrical Engineering and Computer Science, 1997.

[18] Srinivasan, S., Kanagasabapathy, P., and Selvaganesan, N., "Fault Diagnosis in Deaerator Using Neural Networks," Iranian Journal of Electrical and Computer Engineering, Vol. 6, 2007, pp. 62.

[19] De Oliveira, R., and A. T. Marques. "Health monitoring of FRP using acoustic emission and artificial neural networks." Computers \& structures 86, no. 3 (2008): 367-373.

[20] Saxena, A., and Saad, A., "Evolving an Artificial Neural Network Classifier for Condition Monitoring of Rotating Mechanical Systems,” Applied Soft Computing, Vol. 7, 2007, pp. 441-454.

[21] Yang, B. S., Hwang, W. W., Kim D. J., and Chit Tan, A., "Condition Classification of Small Reciprocating Compressor for Refrigerators Using Artificial Neural Networks and Support Vector Machines,” Mechanical Systems and Signal Processing, Vol. 19, 2005, pp. 371-390.

[22] Arel, I., Rose, D. C., and Karnowski, T. P., "Deep Machine Learning - A New Frontier in Artificial Intelligence Research," Computational Intelligence Magazine, IEEE, Vol. 5, No.4, 2010, pp. 13-18.

[23] P. Tamilselvan, P. Wang, B. Youn, "Multi-Sensor Health Diagnosis Using Deep Belief Network based State Classification," ASME 2011 International Design Engineering Technical Conferences (IDETC) and Computers and Information in Engineering Conference (CIE), 2011.

[24] M. Saimurugan, K.I. Ramachandran, V. Sugumaran and N.R. Sakthivel, "Multi component fault diagnosis of rotational mechanical system based on decision tree and support vector machine," Expert Systems With Applications, 2010.

[25] Basudhar, Anirban, and Samy Missoum. "Adaptive explicit decision functions for probabilistic design and optimization 
using support vector machines." Computers \& Structures 86, no. 19 (2008): 1904-1917.

[26] Abbasion, S., Rafsanjani, A., Farshidianfar, A., and Irani, N., "Rolling Element Bearings Multi-Fault Classification Based on the Wavelet Denoising and Support Vector Machine," Mechanical Systems and Signal Processing, Vol. 21, 2007, pp. 2933-2945.

[27] J. Sun, M. Rahman, Y. Wong and G. Hong, "Multiclassification of Tool Wear with Support Vector Machine by Manufacturing Loss Consideration,” International Journal of Machine Tools and Manufacture, Vol. 44, 2004, pp. 1179-1187.

[28] Geramifard, O., Xu, T. X., Pang, C., Zhou, J., and Li, X., "Data-Driven Approaches in Health Condition Monitoring-a Comparative Study," in 8th IEEE International Conference on Control and Automation (ICCA), 2010, pp. 1618-1622.

[29] Wong, M., Jack, L. B., and Nandi, A. K.,"Modified Self-Organising Map for Automated Novelty Detection Applied to Vibration Signal Monitoring,” Mechanical Systems and Signal Processing, Vol. 20, 2006, pp. 593-610.

[30] Field, R. V., and Grigoriu, M., 2006, "Optimal design of sensor networks for vehicle detection, classification, and monitoring," Probabilistic engineering mechanics, 21(4), pp. 305-316.

[31] Guratzsch, R. F., and Mahadevan, S., 2006, "Sensor placement design for SHM under uncertainty," Proc. 3rd European Workshop: Structural Health Monitoring, pp.1168-1175.

[32] Kirkegaard, P., and Brincker, R., 1994, "On the optimal location of sensors for parametric identification of linear structural systems," Mechanical Systems and Signal Processing, 8(6), pp. 639-647.

[33] Udwadia, F., 1994, "Methodology for Optimum Sensor Locations for Parameter Identification in Dynamic Systems," Journal of Engineering Mechanics, 120(2), pp. 368-390.

[34] Azarbayejani, M., El-Osery, A. I., Choi, K. K., and Taha, M. M. R., "A probabilistic approach for optimal sensor allocation in structural health monitoring," Smart Materials and Structures, 17(5), 2008, p. 055019.

[35] Flynn, E. B., and Todd, M. D., 2010, "A Bayesian approach to optimal sensor placement for structural health monitoring with application to active sensing," Mechanical Systems and Signal Processing, 24(4), pp. 891-903.

[36] Ntotsios, E., Christodoulou, K., and Papadimitriou, C., "Optimal sensor location methodology for structural identification and damage detection," Proc. 3rd European Workshop: Structural Health Monitoring, 2006, pp. 1160-1167.

[37] Ikjin Lee, K.K. Choi, Liu Du, David Gorsich, Dimension reduction method for reliability-based robust design optimization, Computers \& Structures, Volume 86, Issues 13-14, July 2008, Pages 1550-1562

[38] Gaofeng Jia, Alexandros A. Taflanidis, Non-parametric stochastic subset optimization for optimal-reliability design problems, Computers \& Structures, Volume 126, 15 September 2013, Pages 86-99

[39] Wang, P., Wang, Z., and Almaktoom, A.T., "Dynamic Reliability-based Robust Design Optimization with Time-Variant Probabilistic Constraints,” Engineering Optimization, 2013. http://dx.doi.org/10.1080/0305215X.2013.79556. 
[40] Alexandros A. Taflanidis, James L. Beck, Stochastic Subset Optimization for reliability optimization and sensitivity analysis in system design, Computers \& Structures, Volume 87, Issues 5-6, March 2009, Pages 318-331

[41] Kurt Maute, Dan M. Frangopol, Reliability-based design of MEMS mechanisms by topology optimization, Computers \& Structures, Volume 81, Issues 8-11, May 2003, Pages 813-824

[42] Dan M Frangopol, Kurt Maute, Life-cycle reliability-based optimization of civil and aerospace structures, Computers \& Structures, Volume 81, Issue 7, April 2003, Pages 397-410

[43] Duan, W.H., Wang, Q., and Quek, S.T., “Applications of Piezoelectric Materials in Structural Health Monitoring and Repair: Selected Research Examples," Materials, 2010, 3(12) 5169-5194.

[44] Kessler, S.S., and Spearing S.M., "Design of a piezoelectric-based structural health monitoring system for damage detection in composite materials," Proc. SPIE 4701, Smart Structures and Materials 2002: Smart Structures and Integrated Systems, 86 (July 12, 2002); doi:10.1117/12.474649.

[45] Zagrai, A., Doyle, D., Gigineishvili, V., Brown, J., Gardenier, H., and Arritt, B., "Piezoelectric Wafer Active Sensor Structural Health Monitoring of Space Structures,” Journal of Intelligent Material Systems and Structures, 2010, 21(9), pp.921-940.

[46] Samuel, Paul, and Darryll J. Pines. "Health monitoring and damage detection of a rotorcraft planetary geartrain system using piezoelectric sensors." In Proceedings of SPIE, vol. 3041, pp. 44-53. 1997.

[47] Dosch, J. J., Inman, D. J., and Garcia, E., 2001, "A self-sensing piezoelectric actuator for collocated control," SPIE milestone series., 167, pp. 319-338.

[48] L. Costa, I. Figueiredo, R. Leal, P. Oliveira, G. Stadler, Modeling and numerical study of actuator and sensor effects for a laminated piezoelectric plate, Computers \& Structures, Volume 85, Issues 7-8, April 2007, Pages 385-403

[49] Schulz, M. J., and M. J. Sundaresan. "Smart Sensor System for Structural Condition Monitoring of Wind Turbines." (2006).

[50] Sérgio L. Schulz, Herbert M. Gomes, Armando M. Awruch, Optimal discrete piezoelectric patch allocation on composite structures for vibration control based on GA and modal LQR, Computers \& Structures, Volume 128, November 2013, Pages 101-115

[51] Sirohi, J., and Chopra, I., "Fundamental Understanding of Piezoelectric Strain Sensors," J. Intell. Mater. Syst. Struct., 2000, 11(4), pp. 246-257.

[52] Marcelo A. Trindade, Ayech Benjeddou, Refined sandwich model for the vibration of beams with embedded shear piezoelectric actuators and sensors, Computers \& Structures, Volume 86, Issue 9, May 2008, Pages 859-869

[53] Henrique Santos, Cristóvão M. Mota Soares, Carlos A. Mota Soares, J.N. Reddy, A finite element model for the analysis of 3D axisymmetric laminated shells with piezoelectric sensors and actuators: Bending and free vibrations, Computers \& Structures, Volume 86, Issue 9, May 2008, Pages 940-947. 
[54] Adjiman, C.S., Androulakis, I.P., and Floudas, C.A., "Global optimization of mixed-integer nonlinear problems,” AIChE J., 2000, 46(9), 1769-1797.

[55] De Maesschalck, R., Jouan-Rimbaud, D., and Massart, D.L. The Mahalanobis distance, Chemometrics and Intelligent Laboratory Systems, 2000, 50, 1-18.

[56] Wei, J., Realff, J., "Sample average approximation methods for stochastic MINLPs,” Comp. Chem. Eng., 2004, 28(3), 333-346.

[57] Lee, S., Youn, B.D., and Jung, B.C., "Robust Segment-Type Energy Harvester and Its Application to a Wireless Sensor," Smart Materials and Structures, 18, 095021(12), 2009. 